http://jmscr.igmpublication.org/home/ ISSN (e)-2347-176x ISSN (p) 2455-0450 crossref DOI: https://dx.doi.org/10.18535/jmscr/v7i10.146

\author{
Journal Of Medical Science And Clinical Research \\ IGM Publication \\ An Official Publication of IGM Publication
}

\title{
Relation between Vitamin D and Severity of Schizophrenia
}

\author{
Authors \\ Shashi Prabha ${ }^{*}$, Pooja Nitin Joshi \\ ${ }^{1}$ Junior Resident, Department of Biochemistry, B.J. Government Medical College, Pune, \\ Maharashtra, India-41100 \\ ${ }^{2}$ Associate Professor, Department of Biochemistry, B.J. Government Medical College, Pune, \\ Maharashtra, India-41100 \\ *Corresponding Author \\ Shashi Prabha
}

\begin{abstract}
Introduction: Schizophrenia is a debilitating psychiatric disorder which is characterised by impairment of thinking, perception, affect and social behaviour. It is one of the leading causes of disability worldwide. The pathophysiology of schizophrenia includes dysregulation of multiple pathways. Vitamin D is implicated in neurodevelopmental origin of schizophrenia. Vitamin D level has been found to be low in patients with mental disorders including schizophrenia.

Objective: 1. To find serum Vitamin D levels in schizophrenic patients.2.To find correlation between Vitamin D level and disease severity based on Positive and Negative Syndrome Scale (PANSS) score.

Method: A Case-control study approved by Institutional Ethics Committee was conducted. The study included 30 cases of schizophrenia and 30 healthy controls based on inclusion and exclusion criteria.

Result: The mean age in years were 36.1 and 35.3 in the case group and control group respectively. The mean vitamin $D$ level in the cases was $11.01 \pm 9.99 \mathrm{ng} / \mathrm{ml}$ and in controls was $18.51 \pm 5.61 \mathrm{ng} / \mathrm{ml}$. This difference was highly significant $(p=<0.001)$. There was a weak negative correlation between vitamin $D$ level in cases and PANSS score $(r=-0.28)$.

Conclusion: Vitamin D levels were low in schizophrenic patients as compared to controls. A weak negative correlation was found between Vitamin D level and severity of disease based on PANSS score. Larger placebo control studies needs to be done to find out potential clinical benefits of Vitamin D supplementation in these patients.
\end{abstract}

Keywords: Vitamin D, schizophrenia, PANSS score.

\section{Introduction}

Schizophrenia is a debilitating mental disorder characterised by impairment of thinking, perception, affect and social behaviour. It is prevalent in all cultures and societies and is a serious health problem. Worldwide the median prevalence of schizophrenia is $4.6 / 1,000$ for point prevalence, 3.3/1,000 for period prevalence, 4.0 for lifetime prevalence, and 7.2 for lifetime morbid risks ${ }^{[1]}$. Both sexes are equally affected but the onset tends to be later in women ${ }^{[2]}$.

Several pathways have been implicated in the pathophysiology of schizophrenia. These include dopaminergic, glutamatergic, GAB Aergic and cholinergic neurotransmitter system $^{[3]}$. Inflammation has been found to play a role in the pathophysiology of schizophrenia ${ }^{[4]}$.

Vitamin D is now considered a potent neuro-steroid hormone and is critical to brain development and 
normal brain function ${ }^{[5],[6]}$. It has several roles in the Central Nervous System. It regulates the production and release of neurotrophic factors such as nerve growth factor (NGF), which is essential for neuron differentiation, regulates synthesis of neuromediators such as acetylcholine ${ }^{[7],[8]}$, regulates expression of genes associated with GABA-ergic neurotransmission [9], regulates catecholamine biosynthesis $^{[10]}$ and maintains cellular calcium homeostasis. Vitamin D is also a neuroprotective which is attributed to its anti-inflammatory effects ${ }^{[11]}$ and its ability to protect against oxidative damage by increasing glutathione levels in neurons ${ }^{12]}$. Vitamin D plays a role in the neurodevelopmental origin of schizophrenia through multiple pathway disruption $^{[13],[14]}$. The various biological pathways in which vitamin D is involved and are also proven to be impaired in schizophrenia are neurotrophic factors, neurotransmission, synaptic and cytoskeleton anomalies, calcium homeostasis, energy metabolism and redox balance ${ }^{[15]}$. These contribute towards the clinical features presented by schizophrenic patients.

The Positive and Negative Syndrome Scale (PANSS) is a medical scale used for measuring symptom severity of patients with schizophrenia. PANSS total minimum score is 30 and maximum is 210 . Higher score indicates more severe disease.

Several studies have been done in the past to assess the role of Vitamin D in Schizophrenia. However, very few studies have been done to find relationship between vitamin D level and disease severity. This study attempts to find this relationship. Several antipsychotic drugs are known to affect calcium homeostasis and might have an effect on Vitamin D levels in patients taking these medications ${ }^{[16]}$. Hence, patients who were not on antipsychotic medications for about 3 months were included in the study.

\section{Aims and Objectives}

1. To find serum Vitamin D levels in schizophrenic patients.

2. To find correlation between Vitamin D level and disease severity based on Positive and Negative Syndrome Scale (PANSS).

\section{Material and Method}

The study was approved by Institutional Ethics Committee. It was a case-control cross-sectional study. The study group included 30 cases and 30 controls with prior informed consent. Cases were the patients with diagnosis of schizophrenia based on International Classification of Diseases (ICD 10) criteria who were not on anti-psychotic medications for the past 3 months. The controls were age and sex matched healthy individuals. The exclusion criteria included: subjects on Vitamin D and Calcium supplementation, subjects with known parathyroid, liver and kidney disorder. Blood sample was collected under strict aseptic conditions. Vitamin D level was estimated by Electro Chemi Luminescence (ECL) analyser (Cobas e411). All the calculations were done using Microsoft Office Excel 2010 and statistical analysis was done using IBM SPSS Statistic version 20. P-value less than 0.05 ( $p<0.05)$ was considered to be statistically significant $(\mathrm{S})$. P-value of less than $0.001(\mathrm{p}<0.001)$ was considered to be statistically highly significant (HS). P-value more than $0.05 \quad(\mathrm{p}>0.05)$ was considered to be statistically non- significant.

\section{Results}

The study included 8 females and 22 males in both cases and controls group.

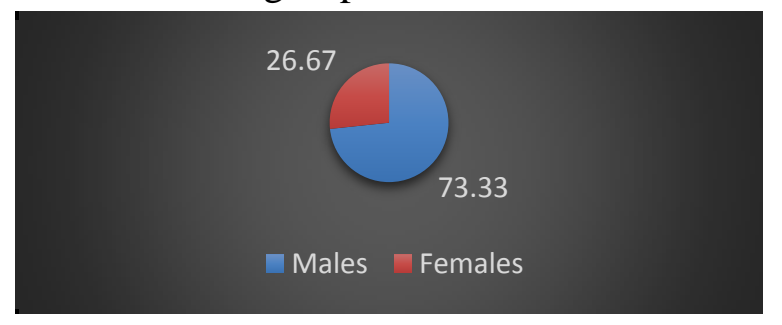

Fig 1: Pie -Diagram showing gender distribution of the study population

The mean age in the Cases group was $36.1 \pm 8.7$ years and in the Controls group was $35.3 \pm 7.4$ years. Table 1: Mean age and Sex Comparison between Patient (Cases) group and Control Group

\begin{tabular}{|l|c|c|}
\hline \multirow{2}{*}{ Gender } & \multicolumn{2}{|c|}{ Groups } \\
\cline { 2 - 3 } & Cases & Control \\
\cline { 2 - 3 } & Mean Age \pm SD & Mean Age \pm SD \\
\hline Male & $36.5 \pm 8.2$ & $36.5 \pm 6.9$ \\
\hline Female & $35 \pm 10.1$ & $32.5 \pm 8.1$ \\
\hline
\end{tabular}




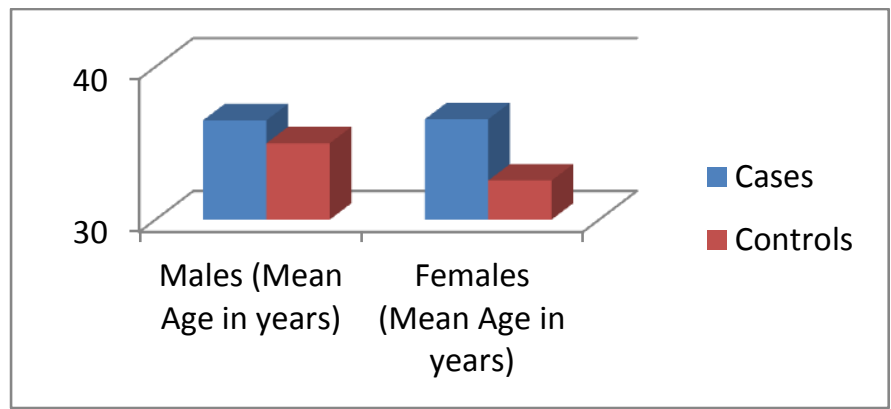

Fig 2: Bar diagram showing age and sex distribution among cases and controls

The difference in age between males and females among the two groups was not significant.

The mean Vitamin D level was $11.01 \pm 9.99$ $\mathrm{ng} / \mathrm{mlin}$ the case group and $18.51 \pm 5.61 \mathrm{ng} / \mathrm{mlin}$ the control group. This difference was highly significant with a $\mathrm{p}<0.001$.

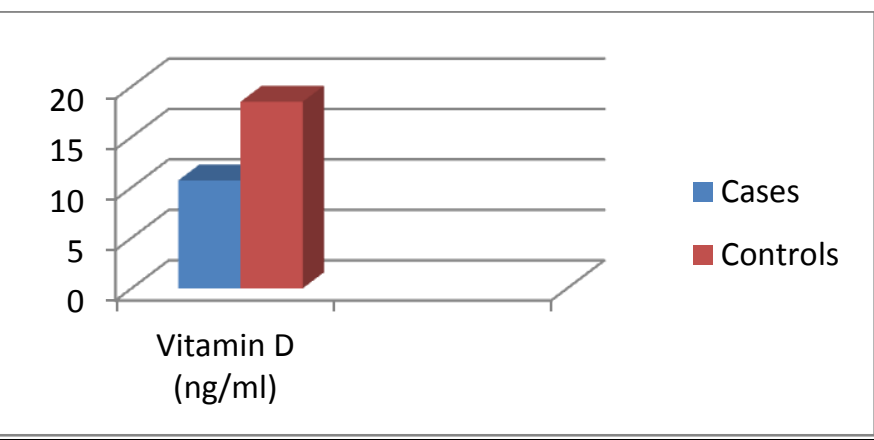

Fig 3: Bar diagram showing mean Vitamin D level (ng/ml) in cases and controls

The severity of disease was assessed using PANSS score. The mean PANSS score of the cases was 74 . A weak, negative but insignificant correlation was found between Vitamin D level in cases and PANSS score $(r=-0.28, p>0.05)$.

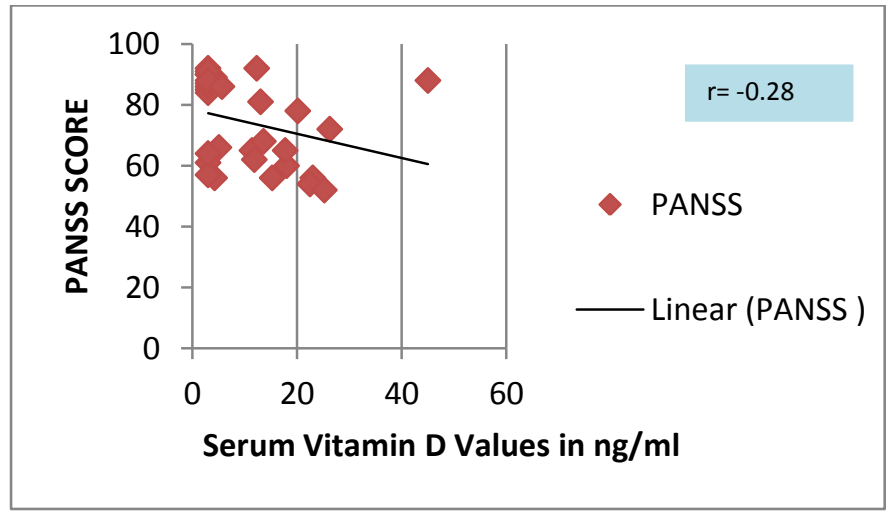

\section{Discussion}

Vitamin D plays an important role in brain development $^{[6]}$ and its deficiency has been implicated in many psychiatric conditions including Schizophrenia. Several studies have been done in the past that point towards the role of developmental deficiency of vitamin D in developing schizophrenia later in life ${ }^{[17]}$

In the present study levels of Vitamin D were low in Schizophrenic cases as compared to controls which support earlier reports ${ }^{[18],[19]}$. This observation could be due to poor nutrition and inadequate sun exposure in patients with psychosis. These patients are at risk of developing complications related to Vitamin D deficiency. A negative correlation between vitamin $\mathrm{D}$ level in patients and disease severity (based on PANSS score) was observed. This finding is not in agreement with the finding of a similar study done by Akinlade et al ${ }^{[19]}$. However, the present study open up avenues for further study on vitamin D level and disease severity as patients with severe disease might be potential candidates for clinical benefits of vitamin D supplementation along with anti-psychotic treatment. Larger placebo control studies needs to be done in future to explore this idea.

\section{Limitation of Study}

The study was very small in terms of sample size and may not reflect the actual population data.

\section{Conclusion}

Vitamin D levels are low in Schizophrenic patients which may affect brain function and this may be one of the contributory factors for clinical symptoms observed in Schizophrenic patients. A negative correlation was found between Vitamin D level and severity of disease based on PANSS score.

\section{References}

1. S. Saha, D Chant, J Welham, J McGrath," A systematic review of the prevalence of schizophrenia”, PLos Med., 2005 May;2(5):e141 
2. ICD-10 Classification of Mental and Behavioural Disorders

3. C Deng, B Dean," Mapping of pathophysiology of schizophrenia: interactions between multiple cellular pathways", Front Cell Neurosci, 2013; 7: 238

4. N Müller," Inflammation in Schizophrenia: Pathogenetic Aspects and Therapeutic Considerations", Schizophr Bull, 2018 Aug; 44(5): 973-982

5. D Eyles, F Feron, X Cui, J Kesby, L Harms, P Ko, J McGrath, T Burne," Developmental vitamin $\mathrm{D}$ deficiency causes abnormal brain development", Psychoneuroendocrinlogy, 2009 Dec;34 Suppl 1:S247-57

6. M Wrzosek, J Łukaszkiewicz, M Wrzosek, A Jakubczyk , H Matsumoto, P Piątkiewicz , M Radziwoń-Zaleska , M Wojnar , G Nowicka ," Vitamin D and the central nervous system",Pharmacol Rep. 2013;65(2):271-8

7. E Garcion, N Wion-Barbot, C MonteroMenei, F Berger, D Wion,"New clues about vitamin D functions in the nervous system", Trends Endocrinol Metab, 2002, 13,100-105

8. J Sonnenberg, V Luine, L Krey, S Christakos,"1,25 Dihydroxyvitamin D3 treatment results in increased choline acetyltransferase activity in specific brain nuclei”, Endocrinology, 1986, 118, 14331439

9. F Féron, T Burne, J Brown, E Smith, J McGrath, A Mackay-Sim, D Eyles," Developmental vitamin D3 deficiency alters the adult rat brain", Brain Res Bull, 2005, 65, 141-148

10. E Puchacz, W Stumpf, E Stachowiak , M Stachowiak," Vitamin D increases expression of the tyrosine hydroxylase gene in adrenal medullary cells", Brain Res Mol Brain Res, 1996, 36, 193-196

11. C Lefebvre d'Hellencourt, C MonteroMenei, R Bernard, D Couez, “ Vitamin D3 inhibits proinflammatory cytokines and nitric oxide production by the EOC13 microglial cell line”, J Neurosci Res, 2003, $71,575-582$

12. L Harms L, T Burne, D Eyles, J McGrath,": Vitamin D and the brain", Best Pract Res Clin Endocrinol Metab, 2011, 25, 657-669

13. J. McGrath, T Burne, F Féron, A MackaySim, DEyles, "Developmental vitamin D deficiency and risk of schizophrenia: a 10year update", Schizophr Bull. 2010 Nov; 36(6): 1073-1078

14. M Chiang, R Natrajan, X Fan," Vitamin D in schizophrenia: a clinical review", Evid Based Ment Health, 2016 Feb;19(1):6-9

15. Ana Dias Amaral, Conceição Calhau, Rui Coelho," Schizophrenia: implications of vitamin D deficit on brain development", International Journal of Clinical Neurosciences and Mental Health

16. D Milovanovic, M Pirkovic, S Simonovic, M Matovic ,S Dejanovic, S Jankovic ,D Ravanic, M Petronijevic, D Risti ,V Mladenovic, M Jovanovic, S Labovic, M Pajovic, D Djokovic, DPetrovic, V Janjic ,"Parameters of Calcium Metabolism Fluctuated during Initiation or Changing of Antipsychotic Drugs" Psychiatry Investig, 2016 Jan; 13(1): 89-101

17. B Samoes, C Silveira," The Role of Vitamin D in the Pathophysiology of Schizophrenia", Neuropsychiatry (2017) Volume 7, Issue 4

18. G Valipour, P Saneei, A Esmaillzadeh," Serum vitamin D levels in relation to schizophrenia: a systematic review and meta-analysis of observational studies", J Clin Endocrinol Metab2014 Oct;99(10):3863-72

19. K Akinlade, O Olaniyan, V Lasebikan, S Rahamon, "Vitamin D Levels in Different Severity Groups of Schizophrenia",Front Psychiatry, 2017 Jun 13;8:105. 